\title{
El estado de la red pública hospitalaria en Colombia para enfrentar el COVID-19, posterior a la política de categorización de riesgo fiscal y financiero*
}

\section{Current situation of the public hospital network in Colombia to face the COVID-19, after the categorization of fiscal and financial risk policy}

\section{0 estado da rede pública hospitalar em Colômbia para atender o COVID-19, após a política de categorização de risco fiscal e financeiro}

Recibido: 11 de Noviembre de 2020. Aceptado: 12 de Marzo de 2021.

Publicado: 30 de diciembre de 2021.

DOI: https://doi.org/10.11144/Javeriana.rgps20.erph

\author{
Gilma Stella Vargas Peña ${ }^{a}$ \\ Universidad de Antioquia, Colombia \\ ORCID: https://orcid.org/0000-0003-4676-0665 \\ Laura Estefany Ruiz Sánchez \\ Metrosalud, Colombia \\ ORCID: https://orcid.org/0000-0002-2126-2451 \\ Luis Alberto Martínez Saldarriaga \\ Asociación de Empresas Sociales del Estado de Antioquia, Colombia \\ ORCID: https://orcid.org/0000-0002-9186-0641
}

Para citar este artículo Vargas GS, Ruiz LE, Martínez LA. El estado de la red pública hospitalaria en Colombia para enfrentar el COVID-19, posterior a la política de categorización de riesgo fiscal y financiero. Rev Gerenc Polit Salud. 2021;20. https://doi.org/10.11144/Javeriana.rgps20.erph

* Artículo de investigación

a Autora de correspondencia. Correo electrónico: gilma.vargas@udea.edu.co 


\section{Resumen}

Problema. El Sistema General de Seguridad Social en Salud basado en políticas neoliberales y en la Nueva Gestión Pública definió políticas de transformación y reestructuración de los hospitales públicos para su declaratoria en riesgo, liquidación/fusión. Posterior a la aplicación de la Política de categorización de riesgo fiscal y financiero (PCRFF) se desconoce el estado de la red pública hospitalaria para enfrentar la pandemia COVID-19. Objetivo. Determinar el estado de la red pública hospitalaria (Empresas Sociales del Estado - ESE) para enfrentar la pandemia COVID-19, luego de la aplicación de la PCRFF. Método. Se realizó un estudio cuantitativo con metodología descriptiva según fuentes secundarias de entidades oficiales. Resultados. Para 2012, de las 968 ESE habilitadas en el país, 657 (67,9\%) estaban en riesgo, y para 2018 fueron 284 (34,1\%). La región Andina aportó el 52,8\% de las ESE en riesgo y 78,3\% de las 23 instituciones liquidadas/fusionadas. De las ESE en riesgo, Antioquia aportó el 16,3\% al país y el 33,3\% a la región Andina y de las ESE liquidadas/fusionadas aportó el 47,8\% al país y el 59\% a la región. Los casos de COVID-19 se concentraron en la región Andina con 65\% de los 3002 037, y en Antioquia con 25\% de los 1968972 de la región Andina. Conclusión. Con la aplicación de la PCRFF se ha limitado la cantidad, el crecimiento y la capacidad de resolución de la red pública hospitalaria, lo que ha afectado la respuesta al Covid-19, de manera armónica y eficiente.

Palabras clave: Riesgo financiero, cierre de hospitales, financiación de la salud, administración financiera de hospitales, infecciones por coronavirus, COVID-19.

\section{Abstract}

Problem. The General System of Social Security in Health, grounded in neoliberal politics and the New Public Management, defined policies of change and restructuration of public hospitals for their risk, liquidation/merger assessment. After the application of the Categorization Policy for Financial and Fiscal Risk (PCRFF, Spanish acronym), the current situation of the public hospital network to face the COVID-19 pandemic is unknown. Objective. To determine the current situation of the public hospital network (Social State Enterprises: ESE, Spanish acronym) to face the COVID-19 pandemic, after the application of the PCRFF. Method. A quantitative study with descriptive methodology was carried out, following secondary sources of official institutions. Results. 657 (67.9\%) out of 968 ESE in 2012 were at risk, and 284 (34.1\%) out of 833 ESE in 2018 were at risk. The Andean region ESE's represented $52.8 \%$ of the risk, and $78.3 \%$ out of the 23 liquidated/merged institutions. Antioquia contributed with $16.3 \%$ of the ESE in risk to the country and $33.3 \%$ to the Andean region; as well as $47.8 \%$ of the liquidated/merged ESE to the country, while $59 \%$ to Andean region. The COVID-19 cases were concentrated in the Andean region with $65 \%$ out of the 3002 037, and Antioquia with 25\% of the 1968972 in the Andean region. Conclusion. With the application of the PCRFF, the quantity, growth and resolution capacity of the public hospital network has been limited, which has affected the response to Covid-19, in a harmonious and efficient way.

Keywords: Financial risk, hospital closure, health financing, hospital financing management, coronavirus infections, COVID-19.

\section{Resumo}

Problema. O Sistema Geral de Segurança Social em Saúde, baseado em políticas neoliberais e na Nova Gestão Pública, definiu políticas de transformação e restruturação dos hospitais públicos para sua declaratória em risco, liquidação/ fusão. Após a aplicação da Política de categorização de risco fiscal e financeiro (PCRFF) se desconhece o estado da rede pública hospitalar para atender a pandemia COVID-19. Objetivo. Determinar o estado da rede pública hospitalar (Empresas Sociais do Estado - ESE) para atender a pandemia COVID-19, após a aplicação da PCRFF. Método. Realizou-se um estudo quantitativo com metodologia descritiva segundo fontes secundárias de entidades oficiais. Resultados. Aplicou-se a PCRFF a 833 ESE em 2018, de 968 ESE em 2012, 657 (67,9\%) estavam em risco e de 833 em 2018, 284 (34,1\%). A Região Andina aportou 150 (52,8\%) em risco e 18 (78,3\%) das 23 liquidadas/fusionadas. Antioquia aportou o 16,3\% de ESE em risco ao país e o 33,3\% à Região Andina e o 47,8\% ao país de 23 liquidadas/ fusionadas, e o 59\% à Região Andina. Os casos de COVID-19 se concentraram na Região Andina com $65 \%$ dos 3 002 037, e em Antioquia com 25\% dos 1968972 na Região Andina. Conclusão. Com a aplicação da PCRFF temse limitado a quantidade, o crescimento e a capacidade de resolução da rede pública hospitalar, o que tem afetado a resposta ao COVID-19, de maneira harmoniosa e eficiente.

Palavras-chave: Risco financeiro, fechamento de hospitais, financiamento da saúde, administração financeira de hospitais, infecções por coronavírus, COVID-19. 


\section{Introducción}

Con los cambios en la economía global y el auge de las políticas de ajuste estructural se dieron transformaciones direccionadas a la reducción del Estado, la descentralización y la privatización (1) apoyadas en la teoría de la Nueva Gestión Pública (NGP). La NGP surgió soportada en ideas económicas y políticas favorables a la gobernanza y al mercado competitivo como alternativa para organizar y gestionar las burocracias menos jerarquizadas y rígidas, para mejorar la calidad, los costos y la competitividad del sector público (2).

Las soluciones de la NGP se basaron en lo que Hood (3) había identificado como un giro drástico en las doctrinas administrativas con predominio de habilidades gerenciales, esquemas de competencia público-privado, pagos variables y estructuras diversas, enfatizando en contratos de prestación de servicios, libertad de gestión y autonomía gerencial, disminuyendo o prescindiendo de reglas que limitaran restricciones a decisiones gubernamentales y presupuestales (4). Se impusieron mecanismos de mercado como: subcontratación, pagos diferenciados, flexibilización de contratos, sistemas de incentivos para el desempeño y sus resultados (5). También, se resalta la preocupación por la calidad, la orientación al cliente/ usuario/ciudadano y la medición de resultados como mecanismo de control, siendo relevantes los convenios de desempeño entre gobierno central y agencias, y entre estas y el personal, cuyo pago o permanencia depende del logro de metas de producción, cobertura, ahorro o satisfacción del usuario (2). Predominan los controles ex post, no ex ante, que aseguren el cumplimiento de estándares: medidas de desempeño, sistemas de monitoreo y control de metas (3).

La NGP no tiene un desarrollo teórico uniforme, pero contempla diversos instrumentos de gestión que fueron aplicados en el sector público en términos del neoliberalismo (2). El Sistema General de Seguridad Social en Salud (SGSSS) en Colombia a partir de 1993 (1) ha estado regido por estos dos modelos y los retos de la globalización, con impacto en la configuración de las políticas sociales y el papel del Estado (6). Bajo este paradigma, a los hospitales públicos se les exigió un nuevo rol como empresas que tenían que competir en el libre mercado, lo que ha desencadenado políticas reduccionistas que los han sometido a cuatro olas de reestructuración, así:

1. Con la Ley 100 de 1993 los hospitales públicos fueron transformados en Empresas Sociales del Estado (ESE), entre 1996-1999, previo cumplimiento de requisitos (7): descentralizadas, con personería jurídica, patrimonio propio y autonomía administrativa. Sus ingresos pasaron del subsidio a la oferta, donde el Estado concurre en la financiación para la prestación de servicios (8), al subsidio a la demanda, o el pago por venta de servicios en un sistema de aseguramiento según contrataciones con administradoras[1] (9), y avales de interventorías-auditorías, $(7,10)$. La salud pasó de ser un bien público a cargo del Estado para convertirse en una mercancía (2). Se favoreció el crecimiento administrativo y los costos de operación, afectando los ingresos y la financiación de las ESE. 
2. El documento Conpes 3204 de 2001-2002 (11) del Consejo Nacional de Política Económica y Social viabilizó la reestructuración de las ESE entre 2002 y 2004, con resultados contractuales flexibilizados y tercerizados a través de cooperativas de trabajo asociado, con procesos previos de indemnización de trabajadores.

3. La política de prestación de servicios (PPS) del 2005 (12) estableció un convenio con el Banco Interamericano de Desarrollo para los procesos de reestructuración. Ambas políticas (Conpes 3204 de 2001-2002 y PPS-2005) tuvieron como objeto la disminución de costos laborales con la tercerización/intermediación. El gobierno nacional sometió a las ESE a convenios de desempeño por 10 años que implicaron pagos a través de créditos condonables, entregados para el financiamiento y sostenimiento, según condiciones de resultados de evaluaciones desde las secretarías departamentales de salud, con apoyo del Ministerio de Hacienda, y que serían retornados ante incumplimientos de condiciones (13). Este sometimiento implicaba para las ESE la restricción en el aumento de su oferta, de sus ingresos, financiación y crecimiento.

4. Con la declaratoria de la emergencia social en salud (2009-2010) se estableció la Política de Categorización de Riesgo Fiscal y Financiero (PCRFF) de las ESE $(13,14)$, que daba los criterios para categorizarlas en riesgo alto, medio, bajo y sin riesgo según su capacidad de financiar las obligaciones operacionales corrientes y no corrientes frente a los ingresos operacionales corrientes (15), es decir, las deudas del año presente y las anteriores se pagan con recursos del año presente producto de recuperación de cartera o pagos de facturación vigente.

La categorización, declarada inexequible en 2011 (16), fue retomada en la Ley 1438 de 2011 (13) y en la Resolución 2509 de 2012 (15) para definir la permanencia de las ESE en el mercado. El Ministerio de Salud y Protección Social (MSPS) emitió ocho resoluciones entre 2012 y 2019 con diferentes criterios de declaratoria de riesgo, donde el Índice de Riesgo se definió sólo con el indicador de Solvencia Financiera, esto es, teniendo en cuenta la capacidad de financiar las obligaciones operacionales corrientes y no corrientes de las instituciones, frente a los ingresos operacionales corrientes (art. 2 Res. 2309 de 2012) (17). Este índice no tiene en cuenta todos los criterios planeados: a) lo presupuestal y financiero, b) la prestación del servicio y c) el mercado; de hecho, estos dos últimos quedaron excluidos. Las variables financiación y permanencia se condicionaron al cumplimiento de los planes de mejoramiento entre ESE y Estado (18). Por otro lado, la Ley 1508 de 2012 (19) proyectó el presupuesto de las ESE teniendo como base solo el recaudo (lo facturado) y no el reconocimiento (lo adeudado por las administradoras), lo que limitó la capacidad de oferta de las ESE por diez años, situación agravada por el sometimiento a carteras morosas acumuladas.

Para Jaramillo (20), previo a la PCRFF los recursos de las ESE eran cada vez más limitados, con afectación en los planes de adquisición de tecnología, la contratación y pagos al talento humano y proveedores. En el mismo sentido, Franco (21) advirtió desde el 2003 la inminencia 
del cierre de hospitales por el creciente déficit financiero y las dificultades administrativas y operativas en toda la red.

La crisis de sostenibilidad del sistema, el debilitamiento y cierre progresivo de ESE y el despojo de derechos laborales de los trabajadores de la salud así como las dificultades administrativas y operativas en toda la red (21) se ha atribuido a las formas de pago (22), a las altas carteras morosas desde las aseguradoras de más de 90 días $(21,23)$ y de casi el 50\% entre 2013 y 2014 $(24,25)$, con sobrecostos en la atención y los retrasos en las obligaciones desde las ESE con sus proveedores (23), por soportar deudas acumuladas de 300 mil millones de pesos en 2000 (21), de $\$ 3,8$ billones de pesos en 2011 (26) y de $\$ 8,2$ billones de pesos, o sea el $60,2 \%$ ( 2 500 millones de dólares) a más de 60 días en 2017 (27). El déficit financiero se atribuyó al alto costo de la intermediación de las aseguradoras (28-30), y en parte, también, al pasivo prestacional no sometido a cálculos actuariales ni a otro tipo de financiación (31,32). Lo anterior ha afectado la capacidad de la oferta, así como la calidad y la accesibilidad a los servicios de salud $(24,25,30,31,33-35)$.

Esta situación no solo ha puesto en riesgo a la red pública hospitalaria, sino a la salud de la población, como bienes públicos, hecho que se hizo evidente cuando la Organización Mundial de la Salud (OMS) declaró el 11 de marzo de 2020 como pandemia al SARS-CoV-2 (36). En Latinoamérica se notificó el primer caso en Brasil el 26 de febrero de 2020, y en Colombia el 06 de marzo de 2020, y el resto de países habían notificado esta infección para el 19 de febrero del mismo año (37).

En Colombia se declaró la emergencia sanitaria el 12 de marzo de 2020 para hacer frente a la pandemia y se generaron políticas para prevenir, tratar y mitigar los efectos económicos, educativos, sociales y de salud $(38,39)$. Frente a esto se generó una gran preocupación por la escasa capacidad instalada y de resolución en la red hospitalaria, especialmente de las ESE y se evidenció que la capacidad de oferta en unidades de cuidado intensivo (UCI) en Colombia, por 100000 habitantes era limitada: de 10,7 (5 349); el número de ventiladores de 11 (5 500) y de especialistas en cuidados intensivos de 2,4 (1 200) (39-42). Para enfrentar el problema se crearon 10 hospitales de campaña y se adecuaron camas de cuidados intermedios como camas de unidades de cuidados intensivos.

En Latinoamérica el aumento sostenido de casos COVID-19 reveló una situación complicada para los hospitales. Para el 31 de marzo de 2021 la ocupación de camas UCI estaba colapsada, presentándose una situación crítica en la región. Así, Paraguay tenía una ocupación del 100\% y pacientes en espera, Perú y Chile del $96 \%$, y Uruguay y Argentina estaban con ocupación de $70 \%$ y $60 \%$ respectivamente (43).

En Colombia, al momento de declaratoria de la emergencia social por COVID-2019 se desconocía el estado de las ESE para enfrentarla, por lo que se planteó determinar el estado de las ESE posterior a la aplicación de la política de categorización de riesgo fiscal y financiero a nivel nacional, regional y local, para enfrentar la pandemia COVID-19. 


\section{Metodología}

Se realizó un estudio cuantitativo con metodología descriptiva, acudiendo a fuentes secundarias de ocho resoluciones sobre PCRFF del MSPS: Resoluciones 2509 de 2012 (15), 1877 de 2013 (44), 2090 de 2014 (45), 1893 de 2015 (46), 2184 de 2016 (47), 1755 de 2017 (48), 2249 de 2018 (49) y 1342 de 2019 (50), y la información suministrada por el MSPS y la extraída de las bases de datos de la Superintendencia Nacional de Salud (Supersalud), de ESE liquidadas entre 2012 y 2018 (51). En 2020 no se emitió Resolución de PCRFF.

La información sobre el COVID-19 se obtuvo en dos momentos: 24 de agosto de 2020 y 07 de mayo de 2021, de las páginas oficiales del MSPS, del Instituto Nacional de Salud (INS) y de diarios colombianos.

Se presentan los resultados del sometimiento de las ESE a la PCRFF y la situación de COVID-19 en Colombia, el estado y preparación de la capacidad instalada hospitalaria para su enfrentamiento, y se hace un análisis de la situación de declaratoria en riesgo de las ESE y de la COVID-19 según regiones y departamentos.

Los datos se tabularon y consolidaron en el programa computacional Microsoft Excel y se analizaron utilizando distribución de frecuencias, porcentajes y análisis de promedios. Se presenta la variación positiva o negativa utilizando el R. (coeficiente de determinación: que indica el porcentaje de variación en $\mathrm{Y}$ explicado por la variación en X). La información se presenta en tablas y figuras.

\section{Hallazgos}

\section{Resultados del sometimiento de las ESE a la PCRFF}

\section{Antecedentes de ESE liquidadas antes de la PCRFF}

Entre 2001 y 2010 fueron liquidadas 13 ESE, dos (15,4\%) de primer y segundo nivel de atención y nueve $(69,2 \%)$ de tercer nivel. De las 13 ESE, siete $(54 \%)$ correspondían a las escindidas del Seguro Social de tercer nivel de atención.

Situación de riesgo de las ESE posterior a la expedición de PCRFF 2012-2018

Al momento de aplicar la PCRFF en 2012 en los 32 departamentos del país había 968 ESE, 311 $(32,1 \%)$ clasificadas sin riesgo y $657(67,9 \%)$ en riesgo. De estas últimas, $414(63 \%)$ estaban en riesgo alto, 127 (19,3\%) riesgo medio y 116 (17,7\%) en riesgo bajo. De las 968 en 2018, $23(2,3 \%)$ estaban liquidadas/fusionadas, quedando 945 con variación negativa de $-2 \%$. No se 
obtuvo información de 135, que incluían 107 no categorizadas por el MSPS, para un total de 833 ESE. De las 833 que quedaban, 527 (63,3\%) estaban sin riesgo y 284 (34,1\%) en riesgo, de las cuales $137(48,2 \%)$ estaban en riesgo alto, $41(14,5 \%)$ en riesgo medio y $106(37,3 \%)$ en riesgo bajo. Ver figura 1.

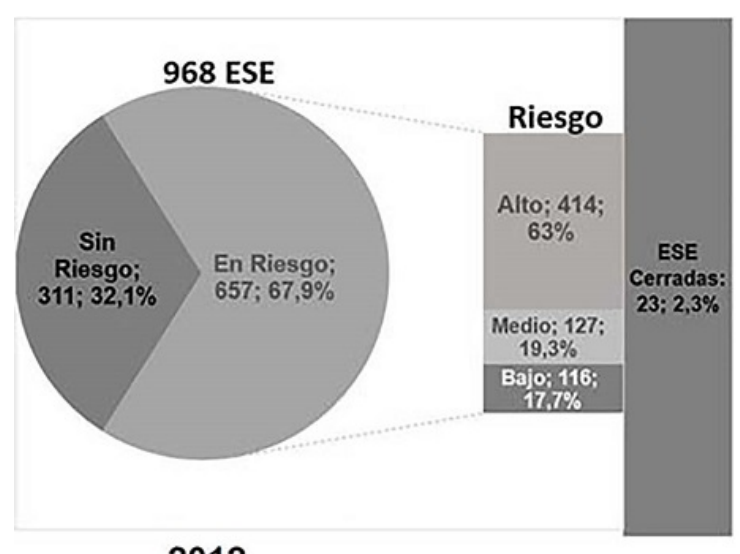

2012

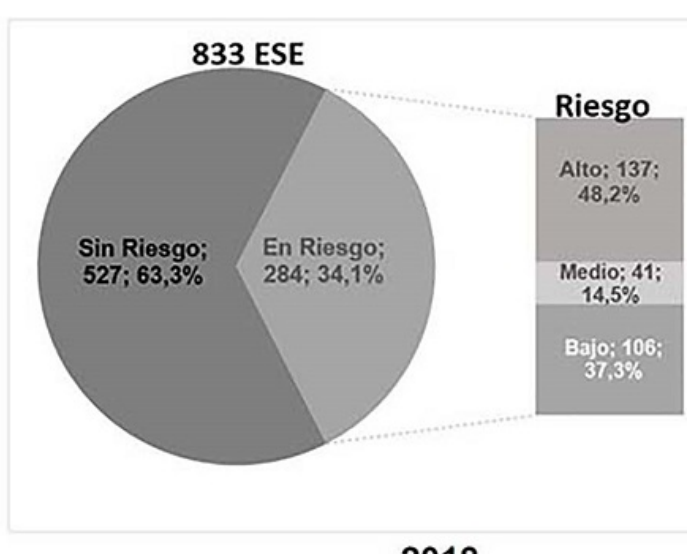

2018

Figura 1 Estado de las ESE según la declaratoria de riesgo del MSPS Colombia 2012 y 2018

Fuente: Resolución 2509 de 2012 y 1342 de 2019, que efectúa la categorización del riesgo de las ESE del nivel territorial, vigencia 2012 y 2018, del MSPS. Base de datos de la Supersalud. Cálculos propios.

\section{Tendencia de la categorización de riesgo financiero de las ESE, 2012-2018}

Según la información del Ministerio de Hacienda y Crédito Público (52), las ESE en riesgo pasaron de $403(41,6 \%)$ en 2012 a $178(19,2 \%)$ en 2018, con una variación negativa de -53,8, y tendencia a disminuir en los últimos tres años: de $28,7 \%$ pasó a $19,7 \%, 21,9 \%$ y a $19,2 \%$. Esta tendencia no fue real pues el MSPS no volvió a categorizar a 185 ESE en 2017, 185 en 2018 y 107 en 2019, que habían sido declaradas en alto o mediano riesgo, asignándoles la misma calificación de riesgo fiscal y financiero del año anterior, omitiendo su esfuerzo para mejorar el indicador. El interés era mantener a las ESE en el programa de saneamiento fiscal y financiero para no incrementar los gastos, a costa del límite de sus ingresos y su crecimiento. Como respuesta a los reclamos de representantes de las ESE, el MSPS las clasificó, pero las excluyó de los indicadores de resultado, entonces a partir de 2017 aparecen como no categorizadas. Para tener información real se realizó un ajuste teniendo en cuenta las no categorizadas con resultados de $39,7 \%$ en $2017,42,2 \%$ en 2018 y $30,8 \%$ en 2019 ESE en riesgo y se observó que de 403 (41,6\%) en riesgo en 2012 pasaron a $285(30,8 \%)$ en 2018, con variación negativa de -26,1, y un R. de 0,227 , es decir, el $22 \%$ de la variación en el riesgo, estuvo influenciada por la variación en los años. La diferencia en los dos resultados es notoria. Ver figura 2. 
Gilma Stella Vargas Peña / Laura Estefany Ruiz Sánchez / Luis Alberto Martínez Saldarriaga

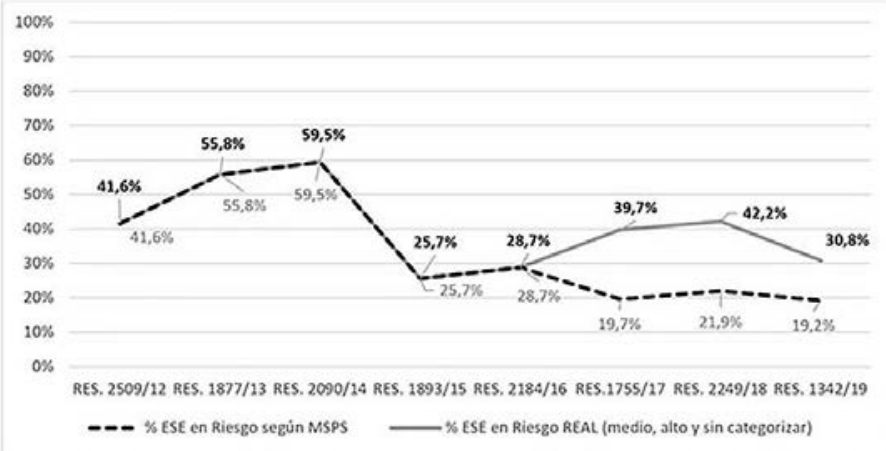

Figura 2 Tendencia de la declaratoria en riesgo de las ESE con y sin las ESE categorizadas por el MSPS, Colombia, 2012-2019

Fuente: cálculos propios con base en la Resoluciones de declaratoria de riesgo de las ESE del MSPS, 2012-2019 y la base de datos de la Supersalud 2012-2018.

Situación de las ESE según la declaración de riesgo por regiones en el 2018

De las 833 ESE en 2018, $284(34,1 \%)$ estaban en riesgo y $527(63,3 \%)$ sin riesgo. De las declaradas en riesgo, $150(52,8 \%)$ fueron de la región Andina, 98 (34,5\%) de la Caribe, 27 $(9,5 \%)$ del Pacífico, 7 (2,5\%) de la Amazonía y 2 (0,7\%) de Orinoquía.

De las 23 ESE fusionadas o liquidadas, 18 (78,3\%) eran de la región Andina, 3 (13\%) de la región Pacífica y $2(8,7 \%)$ del Caribe. Ver tabla 1 . Respecto al total de las ESE en cada región, se observa mayores porcentajes en las regiones Andina y Pacífica.

Tabla 1 Distribución porcentual del estado de las ESE según la declaratoria de riesgo del MSPS, Colombia, 2018

\begin{tabular}{|l|c|c|c|c|c|c|c|c}
\hline \multirow{2}{*}{ Región } & \multirow{2}{*}{$\begin{array}{c}\mathbf{N}^{\circ} \\
\text { Departa- } \\
\text { mentos }\end{array}$} & \multirow{2}{*}{ ESEs } & \multicolumn{2}{|c|}{ En riesgo } & \multicolumn{2}{c|}{ Sin riesgo } & \multicolumn{2}{c}{$\begin{array}{c}\text { Liquidadas/ } \\
\text { fusionadas }\end{array}$} \\
\cline { 5 - 10 } & 11 & 485 & 150 & 52,8 & 318 & $\mathbf{6 0 , 3}$ & 18 & 78,3 \\
\hline Andina & $\mathbf{N ^ { \circ }}$ & $\%$ & $\mathrm{~N}^{\circ}$ & $\%$ & $\mathrm{~N}^{\circ}$ & $\%$ \\
\hline Caribe & 7 & 183 & 98 & 34,5 & 83 & 15,7 & 2 & 8,7 \\
\hline Pacífica & 4 & 129 & 27 & 9,5 & 99 & 18,8 & 3 & 13,0 \\
\hline Orinoquía & 4 & 19 & 2 & 0,7 & 17 & 3,2 & & \\
\hline Amazonia & 6 & 17 & 7 & 2,5 & 10 & 1,9 & & \\
\hline Total & $\mathbf{3 2}$ & $\mathbf{8 3 3}$ & $\mathbf{2 8 4}$ & $\mathbf{1 0 0 , 0}$ & $\mathbf{5 2 7}$ & $\mathbf{1 0 0 , 0}$ & $\mathbf{2 3}$ & $\mathbf{1 0 0 , 0}$ \\
\hline
\end{tabular}

Fuente: cálculos propios con base en la Resolución 1342 de 29 de mayo de 2019 y la base de datos de la Supersalud sobre entidades liquidadas 2012-2018 (29).

Situación de las ESE según la declaración de riesgo por departamentos en 2018

De las 284 ESE en riesgo, 50 (17,6\%) eran de Antioquia, 27 (9,5\%) de Santander, 26 (9,2\%) de Boyacá, 21 (7,4\%) de Sucre, 20 (7,0\%) de Bolívar, 18 (6,3\%) en Atlántico y 17 (6\%) de Magdalena y Cundinamarca respectivamente. Con menor proporción, estuvieron las ESE de 
Tolima (4,9\%), Córdoba (4,6\%) y Valle del Cauca (4,2\%). De estas mismas ESE (284 en algún nivel de riesgo), 249 (87,6\%) eran de primer nivel de atención, 31 (11\%) de segundo y 4 (1,4\%) de tercer.

Las 150 ESE en riesgo en la región Andina se ubicaron en Antioquia (33,3\%), Santander (18\%) y Boyacá (17,3\%); las 98 ESE en la región Caribe correspondieron, principalmente, a Sucre (21,4\%), Bolívar (20,4\%), Atlántico (18,4\%) y Magdalena (17,3\%). De 27 ESE en la región Pacífica la mayoría pertenecían al Valle del Cauca (44,4\%) y Nariño (40,7\%). Para Orinoquía, Arauca y Casanare, una ESE en cada departamento; y siete ESE en Amazonía, Caquetá y Putumayo con 3 ESE (42,9\%) respectivamente, y una en el Amazonas.

La clasificación alto, medio y bajo por departamentos, según total y por regiones, tiene predominio en los departamentos de Antioquia, Cundinamarca y Santander para la región Andina; Atlántico y Bolívar, en la región Caribe, y Nariño y Valle del Cauca, en la región Pacífica. Según el total de ESE en riesgo en la región Andina y en la región Caribe cinco departamentos resaltaron en cada una, encabezados por Antioquia en la primera y Sucre en la segunda. Ver tabla 2.

Tabla 2 Distribución porcentual de la clasificación del riesgo de las ESE según región, departamento y los totales según alto, medio y bajo, Colombia, 2018

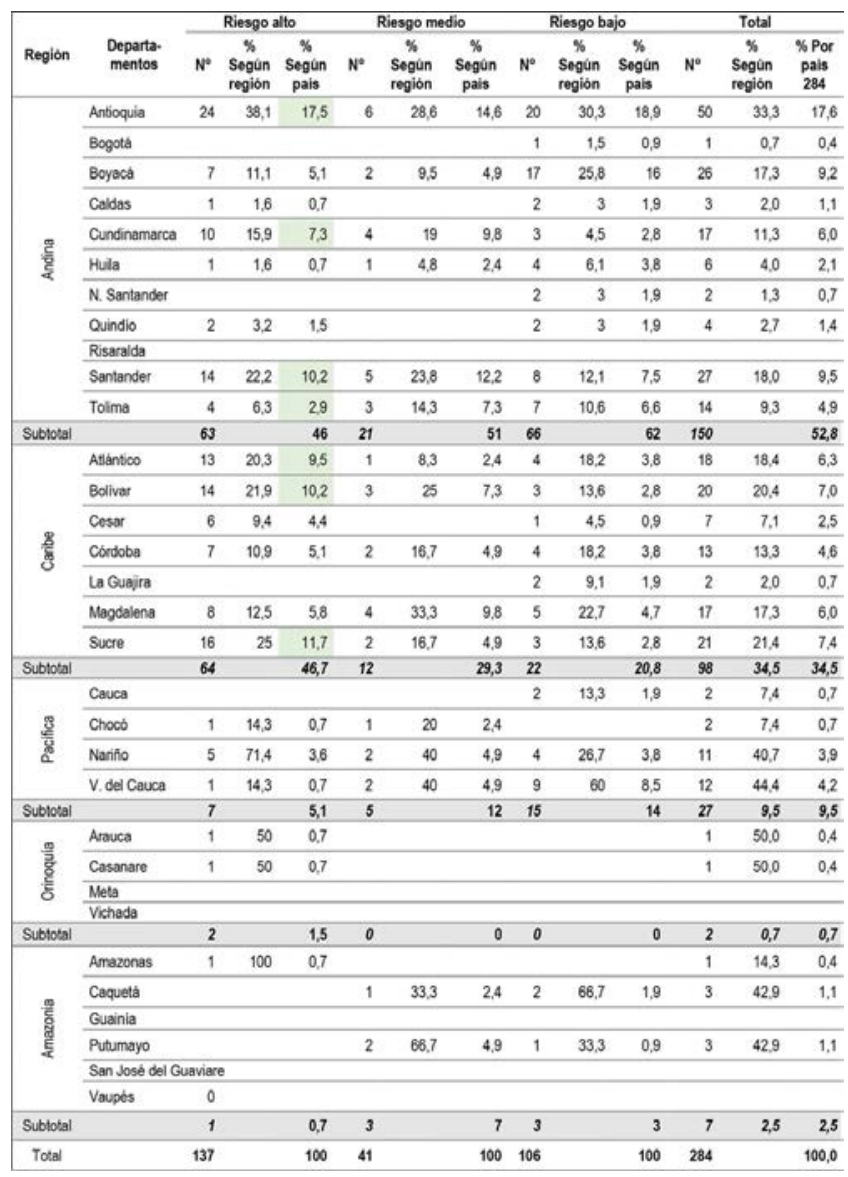

Fuente: Resolución 1342 de 29 de mayo de 2019 del MSPS. Cálculos propios. 


\section{ESE liquidadas/fusionadas por departamento}

Entre 2012 y 2018 fueron liquidadas/fusionadas 23 ESE, de las cuales 18 (78,3\%) eran de la región Andina, 2 (8,7\%) del Caribe y $3(13 \%)$ del Pacífico (ver tabla 1). Del total, 11 (47,8\%) correspondían a Antioquia, $4(17,4 \%)$ a Boyacá, $3(13 \%)$ al Valle del Cauca, $2(8,7 \%)$ a Bolívar, $1(4,3 \%)$ a Caldas, Huila y Santander, respectivamente. Las 18 ESE de la región Andina correspondían a Antioquia (61\%), Boyacá (22,2\%) y Santander, Huila y Caldas con un 5,6\% cada una.

Según el nivel de atención, 17 (73,9\%) eran de primer nivel de atención, 2 (8,6\%) de segundo nivel y $4(17,3 \%)$ de tercer nivel de atención. Una había sido liquidada antes de 2013 y otra en $2013(4,3 \%), 12(52,1 \%)$ en 2014, $4(17,3 \%)$ en 2015, $3(13 \%)$ en 2016 y $2(8,6 \%)$ en 2018.

Al sumar las ESE liquidadas/fusionadas desde 2001, cuando se cerró la primera, el total fue de 36; de las cuales $19(52,7 \%)$ eran de primer nivel de atención, $4(11,1 \%)$ de segundo nivel y $13(36,1 \%)$ de tercer nivel. Las 36 ESE liquidadas/fusionadas correspondieron al 3,6\% de las 981 existentes en 1993.

\section{EI COVID-19 en Colombia}

\section{Tasa de morbimortalidad y letalidad por COVID-19, 2020-2021}

Se tuvieron en cuenta dos fechas de corte con casos acumulados, 24 de agosto de 2020 y 7 de mayo de 2021. De 551698 casos de COVID-19 al 24 de agosto de 2020, pasaron a 3002 037 casos al 7 de mayo de 2021, con crecimiento de 4\%. En este periodo, la proporción de prevalencia acumulada pasó de 1095 a 5959 por 100 mil habitantes con un incremento de 4\%. Los casos recuperados pasaron de 381171 a 2823 672, con variación positiva del 6\%. Las muertes pasaron de 1456 a 77851 , con un aumento del 52\%, y la tasa de mortalidad pasó de 3 a 155 por 100 mil habitantes. La letalidad indica la gravedad de la enfermedad, relacionando muertes y total de enfermos, y hasta el 24 de agosto de 2020 era de 264 por 100 mil habitantes y para el 7 de mayo de 2021 de 2593 por 100 mil habitantes, con un crecimiento de 9\%. Estos indicadores muestran alta propagación de la enfermedad y alta letalidad. El porcentaje de recuperación en los dos cortes fue del 94\% de los casos. Ver tabla 3.

Tabla 3 Tasa de morbimortalidad y letalidad por COVID-19, Colombia, 2020-2021

\begin{tabular}{|l|c|c|c|c|c|c|}
\hline \multicolumn{1}{|c|}{ Fecha } & Enfermos & Recuperados & Muertes & $\begin{array}{c}\text { Tasa } \\
\text { morbilidad }\end{array}$ & $\begin{array}{c}\text { Tasa } \\
\text { mortalidad }\end{array}$ & Letalidad \\
\hline Hasta el 24/08/2021 & 551.696 & 381.171 & 1.456 & 1.095 & 3 & 264 \\
\hline Hasta el 7/05/2021 & 3.002 .037 & 2.823 .672 & 77.851 & 5.959 & 155 & 2.593 \\
\hline \% de variación & & 6 & & 4 & 52 & 9 \\
\hline
\end{tabular}

Fuente: cálculos propios con datos del MSPS. 
En todos los indicadores (enfermos-recuperados-defunciones) la región Andina presentó mayores porcentajes $(65,6 \%, 65,3 \%$ y $60,1 \%)$, la región Caribe tuvo porcentajes entre $19 \%-22 \%$, la región Pacífica entre $11 \%-13,5 \%$, y la Orinoquía y Amazonía con 2\%-2,5\% y 1,3\%-1,7\%, respectivamente. La región Andina resaltó en las tasas por 100 mil habitantes: en morbilidad (7 106), en mortalidad (169) y en letalidad (2 376). La población se proyectó a 2021, ver tabla 4.

Tabla 4 Tasa de morbimortalidad y de letalidad por COVID-19 por 100 mil habitantes según regiones, Colombia, 2020-2021

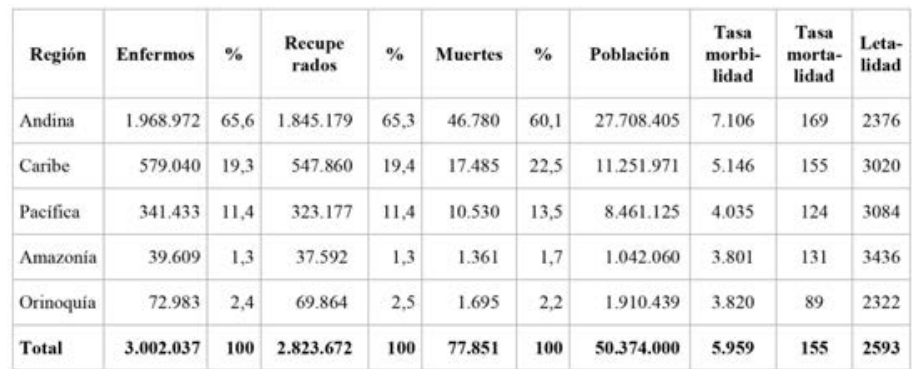

Fuente: cálculos propios con datos del MSPS.

\section{Capacidad hospitalaria para enfrentar el COVID-19}

Según el estudio denominado la Respuesta COVID-19 en América Latina, Colombia reporta 10,7 camas UCI por 100 mil habitantes $(\mathrm{N}=5$ 349), después de Brasil $(\mathrm{N}=26)$, Argentina $(\mathrm{N}=19,2)$ y Chile $(\mathrm{N}=12,1)$. Así mismo, 11 ventiladores por 100 mil habitantes, superado por Brasil (N=31) y Argentina (N=20,4) y 2,4 intensivistas por 100 mil habitantes, igual que Brasil, superado por Argentina (N=3,0) (39).

En Colombia se estipuló que las proyecciones de casos que requerían hospitalización superaban la capacidad instalada de los hospitales del país y que el plan de contingencia del MSPS para ampliar el número de camas UCI en 300\% sería completamente ineficiente. Para la Asociación de Medicina Crítica y Cuidado Intensivo el $80 \%$ de las camas UCI ya estaban ocupadas y solo el $10 \%$ de ellas tenía infraestructura biosegura para el aislamiento, y menos del $2 \%$ tenía presión negativa. La Asociación Colombiana de Empresas Sociales del Estado y Hospitales Públicos se había pronunciado ante el MSPS sobre las dificultades en la red pública hospitalaria para la respuesta oportuna y eficaz del COVID-19 (53).

Según información del Registro Especial de Prestadores de Servicios de Salud (REPS) del MSPS, ningún departamento tenía más de dos camas UCI por cada 100.000 habitantes, y eran inexistente en aquellos con densidad poblacional baja y rural dispersa (Ej.: Vaupés, Vichada, Guainía, Amazonas y Guaviare) (54). En el mismo sentido, las camas de Unidades de Cuidados Especiales ya estaban al 90\% de su ocupación (55).

Para solucionar la escasa capacidad instalada y atender casos de COVID-19 se planteó la apertura de Instituciones Prestadoras de Servicios de salud de las administradoras, así como 
de otras ESE previamente cerradas y la proyección de hospitales de campaña. Hubo una respuesta específica por ciudades, por ejemplo, en Barranquilla se propuso la adecuación de coliseos (baloncesto-boxeo) y la oferta de instalaciones de seis hoteles para 400 habitaciones. En Cali se proyectaron 800 camas con reapertura de dos clínicas liquidadas de SaludcoopEPS y el monitoreo de 500 pacientes en hoteles. En Bogotá abrieron más de 900 camas de baja complejidad en Corferias y se hizo reapertura y habilitación de mil camas de hospitales liquidados de Saludcoop, y se adecuaron diez hospitales de campaña (53). También se abrieron ESE previamente liquidadas, como en Mompós, Bolívar, una de segundo nivel de atención para más de 234000 personas del municipio y de otros del departamento. O en Envigado, Antioquia, la ESE Santa Gertrudis de primer nivel de atención con 38 camas dotadas como apoyo al hospital de segundo nivel de atención (56).

\section{La situación de las ESE y de COVID-19 según regiones y departamentos}

La región Andina, aquella con más casos de COVID-19 del país (el 65,6\%), ha sido también la más afectada con la PCRFF, con 52,8\% del total de sus ESE en riesgo y el 78,3\% de las liquidadas/fusionadas del país. Le sigue la región Caribe con 19,3\% de casos COVID-19 y el 34,5\% de ESE en riesgo y el 8,7\% de las liquidadas/fusionadas. La región Pacífica con 11,4\% casos de COVID-19, el 9,5\% de las ESE en riesgo y el 13\% de las liquidadas/fusionadas. Estas regiones aportaron el 95\% casos COVID-19, así como el 96,3\% de las ESE en riesgo y el 100\% de las liquidadas/fusionadas.

Por departamentos, Antioquia aportó el 16,3\% de casos de COVID-19 del país (25\% región en la Andina), el 17,6\% de las ESE en riesgo (33,3\% en la región Andina), y el 47,8\% de las liquidadas/fusionadas (61\% en la región Andina). Bogotá tuvo el 27,7\% de los casos de COVID-19 y el 0,4\% de las ESE en riesgo. En el mismo orden de indicadores, Santander tuvo el 3,7\%, 9,5\% y 4,3\%, y Boyacá el 1,9\%, 9,5\% y 17,4\% de las ESE fusionadas/liquidadas. De la región Caribe, Bolívar con 2,9\%, 7\% y 8,7\%, y de la región Pacífica, el Valle del Cauca con $8,2 \%, 4,2 \%$ y $13 \%$. Ver tabla 5 . 
Tabla 5 Distribución porcentual de las ESE clasificadas en riesgo y liquidadas/ fusionadas, y de casos de COVID-19, según región y departamento, Colombia, 2021

\begin{tabular}{|c|c|c|c|c|c|c|c|c|c|c|}
\hline 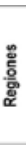 & $\begin{array}{c}\text { Departamen- } \\
\text { tos }\end{array}$ & $\begin{array}{l}\text { ESE en } \\
\text { riesgo }\end{array}$ & $\begin{array}{l}\text { ESE } \\
\text { liquida- } \\
\text { das/ } \\
\text { fusiona- } \\
\text { das }\end{array}$ & $\begin{array}{l}\text { Casos } \\
\text { COVID } \\
\\
(6603 / 20 . \\
7105 / 21)\end{array}$ & $\begin{array}{l}\text { \% ESE en } \\
\text { riesgo } \\
\text { Atotal } \\
\text { región }\end{array}$ & $\begin{array}{c}\% \text { ESE } \\
\text { en } \\
\text { riesgol } \\
\text { total } \\
\text { pais }\end{array}$ & $\begin{array}{c}\text { \% ESE } \\
\text { liquidadas } \\
\circ{ }^{\circ} \\
\text { fusionadas/ } \\
\text { total región }\end{array}$ & $\begin{array}{l}\text { \% ESE } \\
\text { liquida- } \\
\text { das o } \\
\text { fusiona- } \\
\text { das/ } \\
\text { total } \\
\text { pais }\end{array}$ & $\begin{array}{l}\% \text { casos } \\
\text { Covid-19/ } \\
\text { total } \\
\text { región }\end{array}$ & $\begin{array}{c}\text { \% Casos } \\
\text { Covid-19/ } \\
\text { total } \\
\text { casos } \\
(3.002 .037)\end{array}$ \\
\hline \multirow{12}{*}{$\frac{\pi}{8}$} & Antioquia & 50 & 11 & 490.301 & 33,3 & 17,6 & 61 & 47,8 & 25 & 16,3 \\
\hline & Santander & 27 & 1 & 109.596 & 18 & 9,5 & 6 & 4.3 & 5,6 & 3,7 \\
\hline & Boyaca & 26 & 4 & 57.044 & 17,3 & 9.2 & 22 & 17,4 & 3 & 1.9 \\
\hline & Cundinamarca & 17 & & 134.917 & 11,3 & 6.0 & & & 7 & 4.5 \\
\hline & Tolima & 14 & & 71.002 & 9,3 & 4,9 & & & 4 & 2,4 \\
\hline & Hulia & 6 & 1 & 54.750 & 4 & 2,1 & 6 & 4.3 & 3 & 1,8 \\
\hline & Quindio & 4 & & 40.652 & 2,7 & 1,4 & & & 2 & 1,4 \\
\hline & Caldas & 3 & 1 & 64.358 & 2 & 1,1 & 6 & 4,3 & 3 & 2,1 \\
\hline & $\begin{array}{l}\text { Norte } \\
\text { Santander }\end{array}$ & 2 & & 56.309 & 1.3 & 0.7 & & & 3 & 1.9 \\
\hline & Bogota & 1 & & 831.252 & 0,7 & 0.4 & & & 42 & 27,7 \\
\hline & Risaralda & & & 58.791 & & & & & 3 & 2.0 \\
\hline & Subtotal & 150 & 18 & 1.968.972 & 100 & & 100 & & 100 & \\
\hline & $\begin{array}{l}\text { \% segün el gran } \\
\text { total }\end{array}$ & 52,8 & 78,3 & 65,6 & & 18,6 & & & & \\
\hline \multirow{9}{*}{ 행 } & Sucre & 21 & & 30.298 & 21,4 & 7,4 & & & 5 & 1.0 \\
\hline & Bolivar & 20 & 2 & 86.748 & 20,4 & 7,0 & 100 & 8,7 & 15 & 2.9 \\
\hline & Atlántico & 18 & & 249.074 & 18,4 & 6.3 & & & 43 & 8.3 \\
\hline & Magdolona & 17 & & 62.733 & 17,3 & 6,0 & & & 11 & 2,1 \\
\hline & Cordoba & 13 & & 55.962 & 13,3 & 4.6 & & & 10 & 1,9 \\
\hline & Cesar & 7 & & 57.883 & 7,1 & 2.5 & & & 10 & 1.9 \\
\hline & La Guajira & 2 & & 32.258 & 2 & 0.7 & & & 6 & 1,1 \\
\hline & $\begin{array}{l}\text { San Andrés y } \\
\text { Providencia }\end{array}$ & & & 4.084 & & & & & 1 & 0,1 \\
\hline & Subtotal & 98 & 2 & 579.040 & 100 & & 100 & & 100 & \\
\hline & $\begin{array}{l}\text { 7seguin el gran } \\
\text { total }\end{array}$ & 34,5 & 8,7 & 19,3 & & & & & & \\
\hline \multirow[b]{6}{*}{$\%$} & $\begin{array}{l}\text { Valle. del } \\
\text { Cauca }\end{array}$ & 12 & 3 & 245.340 & 44,4 & 4,2 & 100 & 13,0 & 72 & 8.2 \\
\hline & Nariño & 11 & & 55.796 & 40,7 & 3.9 & & & 16 & 1.9 \\
\hline & Cauca & 2 & & 31.384 & 7,4 & 0,7 & & & 9 & 1,0 \\
\hline & Choos & 2 & & 8.913 & 7,4 & 0,7 & & & 3 & 0,3 \\
\hline & Subtotal & 27 & 3 & 341.433 & 100 & & 100 & & 100 & \\
\hline & $\begin{array}{l}\text { 6s seguin el gran } \\
\text { total }\end{array}$ & 9,5 & 13,0 & 11,4 & & & & & 0 & \\
\hline
\end{tabular}

Fuente: Resolución 1342 del 29 de mayo de 2019 del MSPS. INS, casos de COVID-19 al 7 de mayo de 2021.

\section{Discusión}

En consonancia con las políticas neoliberales y la NGP se aplicó la PCRFF que clasifica a las ESE en riesgo y las obliga a proyectar su presupuesto según recaudo (lo facturado) y no el reconocimiento (lo adeudado por las administradoras). El resultado en 2018 fue 284 $(34,1 \%)$ ESE en riesgo y 23 liquidadas, con mayor representatividad de la región Andina y en el departamento de Antioquia, y con implicaciones importantes en las de primer y tercer nivel de atención.

Por lo general, las ESE han estado sometidas a retrasos en los pagos y a carteras morosas de más de 180 días. En 2017 la Asociación Colombiana de Hospitales y Clínicas (27) encontró deudas, desde las Administradoras hacia las ESE, por $\$ 8,2$ billones y carteras morosas mayores de 60 días en el 60,2\% (2 500 millones de dólares). Al 31 de diciembre de 2020 las deudas superaban 9042 billones de pesos (57), afectando las finanzas de más de 900 ESE, y con el 60\% de deuda con mora superior a 90 días, comprometiendo su funcionamiento y permanencia (58). Ya Jaramillo (20) desde 2001 había alertado al respecto. El sometimiento de la ESE a permanentes categorizaciones de riesgo y procesos de liquidación/fusión y privatización ha exacerbado la crisis hospitalaria, lo que favorece el pensamiento rentista y atenta contra el derecho a la salud de la población $(24,25,30,33,34,59-64)$. 
Las ESE están atrapadas en el círculo de carteras morosas que limitan la solvencia financiera, favorece su declaratoria en riesgo y su sometimiento a convenios de desempeño, a realizar presupuestos según el recaudo, mas no el reconocimiento, y limita su desarrollo y crecimiento por diez años, para finalmente ser liquidadas/fusionadas. La situación es alarmante para garantizar la oferta, puesto que la mayoría de las ESE de primer nivel de atención funcionan como un monopolio, en áreas con poca densidad o alta ruralidad y, concentradas principalmente en la región Andina y el Caribe, que concentran el 63\% de la población, según el DANE $(65,66)$. Esta situación ha afectado la capacidad de resolución de las ESE de primer nivel de atención, obligando la derivación de atenciones de baja severidad a instituciones de segundo y tercer nivel de atención públicas o privadas, contribuyendo al morbicentrismo concentrado en la alta complejidad. Según Escobar (24) el propósito del modelo neoliberal es la disminución del gasto público y el fomento de la medicina biologista, en detrimento de una holista o biopsicosocial.

Se ha limitado la operación armónica de la red de servicios de salud haciéndola más ineficiente y costosa para el sistema, lo que contradice lo planteado en el informe Dawson, que el 80\% de los problemas de salud se resuelven en el primer nivel de atención, el 15\% en el segundo nivel y el 5\% en el tercero (67). Esta situación, sumada a otras dificultades evidenciadas en la red pública hospitalaria (53), se evidenció al momento de enfrentar la pandemia COVID-19 en el país, que obligó a enfrentar la escasez de camas UCI o la inexistencia de estas en muchas regiones $(51,39)$, y la alta ocupación por otras patologías (55). Los esfuerzos se orientaron a la dotación con camas UCI, a abrir ESE previamente liquidadas, y a adecuar espacios como hospitales de campaña en las grandes ciudades, principalmente. La problemática se concentra en la región Andina -en particular en el Departamento de Antioquia- que es la que más casos de COVID-19 ha aportado al país (65,6\%), y la que ha sido más afectada con la PCRFF.

La atención del COVID-19 se centró en el tercer nivel de atención, desconociendo la importancia del primer nivel en la red de servicios de salud, que apoyados en la APS renovada (68) pudieran brindar mejores respuestas, con modelos orientados a la educación en salud y para la salud, como parte de la promoción de la salud y prevención de la enfermedad, como lo plantea el Modelo de Acción Integral Territorial (69), teniendo en cuenta que los problemas de salud son locales y se deben resolver en y con las comunidades.

\section{Conclusión}

La aplicación de la PCRFF, fundamentada en el neoliberalismo y en la NGP, y la declaratoria de riesgo y liquidación/fusión a las ESE, devino en 2012 en que 657 (67,9\%) de las 968 activas, fueran categorizadas en riesgo, y 23 (2,3\%) liquidadas/fusionadas. Al inicio de la pandemia COVID-19, había 833 ESE y $284(34,1 \%)$ en riesgo. La región Andina ha tenido que soportar el 65,6\% de 3002037 casos de COVID-19 del país, el 52,8\% de las ESE en riesgo y el 78,3\% de las 23 liquidadas/fusionadas. Y Antioquia, que ha aportado el 16,3\% del total de casos de COVID-19 del país y el 25\% de los casos de la región Andina, tiene el 17,6\% de las 284 ESE en riesgo del país y el 33,3\% de 150 en riesgo en la región Andina, y el 47,8\% de las 23 ESE 
liquidadas/fusionadas del país, así como el 61\% de las 18 liquidadas/fusionadas de la región Andina.

En ese sentido, la aplicación de la PCRFF ha limitado la oferta de servicios de salud en la figura de las ESE, al momento de enfrentar la pandemia por COVID-19. Surge, entonces, la necesidad de rescatar y potenciar la red pública hospitalaria definiendo otros parámetros de medición y que den cuenta de su rentabilidad social más que de la rentabilidad financiera.

\section{Consideraciones éticas}

El artículo es producto de la Tesis doctoral: "Comportamiento y relación de los indicadores de las áreas funcionales de las ESE entre 2017-2011 y a partir de la política de reestructuración hospitalaria. Departamento de Antioquia" que fue clasificada como sin riesgo y fue aprobada por el Comité de Investigación de la Facultad Nacional de Salud Pública de la Universidad de Antioquia, en el Acta de Registro y Propiedad Intelectual 472 del 21 de febrero de 2019.

\section{Contribución de los autores}

G. S. Vargas participó en el procesamiento, elaboración del plan de análisis y análisis de la información, y lideró y participo en la elaboración del artículo. L. Ruiz participó en la búsqueda de la información, tabulación y consolidación, así como en la elaboración del artículo. L. A. Martínez participó en la elaboración del artículo.

\section{Financiación}

El artículo es producto de una tesis doctoral que contó con el apoyo económico del Grupo de Investigación Gestión y Políticas de Salud, a través del Centro de Investigación de la Facultad Nacional de Salud Pública INV 629-18.

\section{Conflictos de interés}

Este artículo se proyecta ser presentado en eventos académicos nacional o internacional. Los autores declaran no tener conflicto de interés que impida o limite la presentación de la investigación.

\section{Referencias}

1. Burki S, Perry G, Dillinger W. Estudios del Banco Mundial sobre América Latina y el Caribe: más allá de centro: la descentralización del Estado. Washington D.C.: Banco Mundial; 1999.

2. Cejudo G. Nueva Gestión Pública. México: Siglo XXI editores; 2011.

3. Hood C. A public management for all seasons? Rev. Public Administration. 1991; 69(1):3-19. http://ne wdoc.nccu.edu.tw/teasyllabus/110041265941/Hood\%20NPM\%201991.pdf 
4. Thomas J, Robert H. In Search of Excellence: Lessons from America's. Best-Run Companies. Nueva York: Harper \& Row; 1982.

5. Dunleavy P. Democracy, Bureaucracy and Public Choice. Economic Explanations in Political Science. Nueva York: Harvester Wheatsheaf; 1991.

6. Guerra DE. El neoliberalismo como amenaza para el acceso a la salud de los colombianos. Rev Fac Nac Salud Pública. 2006;24(2):142-146. http://www.scielo.org.co/pdf/rfnsp/v24n2/v24n2a14.pdf

7. Colombia. Congreso de la República. Ley 100 de 1993 por la cual se crea el sistema de seguridad social integral y se dictan otras disposiciones. Diario Oficial, 41146 (Dic. 23 1993).

8. Colombia. Congreso de la República. Ley 1955 de 2019, mayo 25, por el cual se expide el plan nacional de desarrollo 2018-2022 Pacto por Colombia, pacto por la equidad. Bogotá; El Congreso.

9. Gómez LA. La crisis hospitalaria: análisis de la producción y el pago por servicios individuales de salud en un hospital de primer nivel de atención de la res publica de Bogotá. Rev Salud Bosque. 2012;2(2):61-68. https://revistas.unbosque.edu.co/index.php/RSB/article/view/64/50

10. Reyes S, Ruiz F, Acosta N, Eslava JI, Puente CA, Ardila Z y Peñalosa E. Entorno, aseguramiento y acceso en el régimen subsidiado en Colombia: Seis casos de acceso. Santafé de Bogotá: Fundación Corona y Cendex; 1999.

11. DNP - Colombia. Documento Conpes 3204. Política de prestación de servicios para el sistema de seguridad social en salud y asignación de recursos del presupuesto general de la nación para la modernización de los hospitales públicos. https://colaboracion.dnp.gov.co/CDT/Conpes/Econ\%C3\% B3micos/3204.pdf

12. Colombia. Minsalud. Política Nacional de Prestación de Servicios de Salud [Internet] https://www.minsalud.gov.co/Ministerio/Documents/Politica\%20Nacional\%20de\%20Pre staci $\%$ C3\%B3n\%20de\%20Servicios\%20de\%20Salud.pdf

13. Colombia. Congreso de la República. Ley 1438 de 2011, enero 19, por medio de la cual se reforma el Sistema General de Seguridad Social en Salud y se dictan otras disposiciones. Art 80 y 81. Bogotá: El Congreso; 2011.

14. Colombia. Ministerio de la Protección Social. Decreto 4975 de 2009, diciembre 23, por el cual se declara el Estado de Emergencia Social. Bogotá: El Ministerio; 2009.

15. Colombia. Ministerio de Salud y Protección Social. Resolución 2509 por medio de la cual se define la metodología para la categorización del riesgo de las Empresas Sociales del Estado del nivel territorial y se efectúa la categorización del riesgo para la vigencia 2012. Diario Oficial, 48538 (Ago. 302012 ).

16. Colombia. Corte Constitucional. Sentencia C- 156 de 2011, marzo 9, Revisión de constitucionalidad del Decreto 4580 de 7 de diciembre de 2010. Bogotá: La Corte; 2011.

17. Serrano J, Villareal J, García O. Administración financiera, fundamentos y aplicaciones. McGrawHill, Prensa Moderna Impresores S.A, $3^{\text {a }}$ edición, 1999.

18. Villar LA. La Ley 100: el fracaso estatal en la salud pública. Deslinde. 2009;36:1-10. https://cedetrab ajo.org/wp-content/uploads/2012/08/36-14.pdf 
19. Colombia. Congreso de la Republica Ley 15082012 por la cual se establece el régimen jurídico de las Asociaciones Público-Privadas, se dictan normas orgánicas de presupuesto y se dictan otras disposiciones. Diario Oficial, 48308 (Ene. 10 2012).

20. Jaramillo I. El Nuevo Papel del Estado en la Prestación y Aseguramiento de los Servicios de Salud. Rev Salud Pública. 2001;3(3):191-222. https://iris.paho.org/bitstream/handle/10665.2/53125/978927532 2659_spa.pdf?sequence $=1 \&$ is Allowed $=\mathrm{y}$

21. Franco S. Para que la salud sea pública: Algunas lecciones de la Reforma de Salud y Seguridad Social en Colombia. Rev Gerenc Polit Salud. 2003;2(4):58-70. https://revistas.javeriana.edu.co/index.php/ gerepolsal/article/view/2805

22. Londoño B, Jaramillo I, Uribe J. Descentralización y reforma en los servicios de salud: El caso colombiano. Bogotá: Banco Mundial; 1999.

23. Molina G, Vargas J, Berrío A, Muñoz DP. Características de la contratación entre aseguradores y prestadores de servicios de salud, Medellín, 2007-2008. Rev Gerenc Polit Salud. 2010;9(18):103-15. https://doi.org/10.11144/Javeriana.rgsp9-18.ccea

24. Escobar J. Obstinación jurídica: el caso de la Ley 100 de 1993. Rev Colomb Bioét. 2013;8(2):129-139. https://doi.org/10.18270/rcb.v8i2.796

25. Gómez RD, Nieto E. Colombia: ¿Qué ha pasado con su reforma de salud? Rev. Peru Med Exp Salud Publica. 2014;31(4):733-739. http://www.scielo.org.pe/pdf/rins/v31n4/a19v31n4.pdf

26. Cadena L, Aldana B. Los profesionales de la salud y la crisis del sistema de salud en Colombia: ¿indignación o acomodamiento? MedUNAB. 2011;14(2):83-85. https://revistas.unab.edu.co/index.p $\mathrm{hp} /$ medunab/article/view/1558/1465

27. Asociación Colombiana de Hospitales y Clínicas. La realidad financiera de los hospitales y clínicas. ht tp://achc.org.co/wp-content/uploads/2018/01/Realidad-Financiera-Hospitales.pdf

28. Gorbaneff Y, Torres S, Contreras N. Anatomía de la cadena de prestación de salud en Colombia en el régimen contributivo. INNOVAR, revista de ciencias administrativas y sociales. 2004;23:168-181. h ttp://www.scielo.org.co/pdf/inno/v14n23/v14n23a13.pdf

29. Ahumada C. La penuria de la salud pública. Rev Gerenc Polit Salud. 2002;1(3):47-56. https://revistas .javeriana.edu.co/index.php/gerepolsal/article/view/2882

30. Tobón F. ¿Eliminar la intermediación de la EPS para asignarle esta función al Estado? ¡Peor el remedio que la enfermedad! Perf. de Coyunt. Econ. 2012;19(1):61-75. https://revistas.udea.edu.co/index.php /coyuntura/article/view/15553/13488

31. Cárdenas M, Velasco BM. Incidencia de la morosidad de las cuentas por cobrar en la rentabilidad y la liquidez: estudio de caso de una Empresa Social del Estado prestadora de servicios de salud. Rev Fac Nac Salud Pública. 2014;32(1):16-25. http://www.scielo.org.co/pdf/rfnsp/v32n1/v32n1a03.pdf

32. Colombia. Presidencia de la República. Decreto 306 de 2004, por el cual se reglamentan los artículos 61, 62 y 63 de la Ley 715 de 2001. Diario oficial, 45450 (Feb. 3 2004).

33. Eslava JC. Hospital Universitario y crisis hospitalaria en Colombia. Rev Gerenc Polit Salud. 2002;1(2):41-48. https://www.redalyc.org/pdf/545/54510205.pdf 
34. Moreno MC, López MV. La salud como derecho en Colombia. 1999-2007. Rev Gerenc Polit Salud. 2009;8(16):133-152. https://www.redalyc.org/articulo.oa?id=54514071008

35. Echeverry ME, Borrero YE. Protestas sociales por la salud en Colombia: la lucha por el derecho fundamental a la salud, 1994-2010. Cad Saúde Pública. 2015;31(2):354-364. https://www.scielo.br/ j/csp/a/zX4Jj45XrgGf5Yv4jg8WhtK/abstract/?lang=es

36. Organización Mundial de la salud. Alocución de apertura del Director General de la OMS en la rueda de prensa sobre la COVID-19 celebrada el 11 de marzo de 2020. [Consultado 2020 Ago 1] https://www.who.int/es/dg/speeches/detail/who-director-general-s-opening-remarks-at-the-media -briefing-on-covid-19---11-march-2020

37. Johns Hopkins University. Mapa COVID-19. https://coronavirus.jhu.edu/map.html]

38. Colombia. Ministerio de Salud y Protección Social. Resolución 385 de 2020, marzo 12, por la cual se declara la emergencia sanitaria por causa del coronavirus COVID-19 y se adoptan medidas para hacer frente al virus. Bogotá: El Ministerio; 2020.

39. García P, Alarcón A, Bayer A, Buss P, Guerra G, Riveiro H et al. COVID-19 Response in Latin America. Am. J. Trop. Med. Hyg. 2020;00:1-8. http://www.ajtmh.org/docserver/fulltext/10.4269/ajtmh.20-0765/tpmd200765.pdf?exp ires $=1604370859 \& \mathrm{id}=\mathrm{id} \&$ accname $=$ guest $\&$ checksum=E59F7DB5E7F903C5B66FAA64D10EFB4 0

40. Unidad de datos del El Tiempo. ¿Cuántas camas de UCI por persona hay en Colombia? El Tiempo. 2020 Mar 28 [Consultado 2020 Sep 21] Disponible en: https://www.eltiempo.com/datos/total-de-camas-d e-cuidado-intensivo-en-colombia-478076

41. Accini JL. Existe en Colombia la necesidad para gestionar una ley que reglamente la prestación de servicios de cuidados intensivos? Cuáles son sus alcances e implicaciones potenciales? A.C.C.I. 2017;17(1):1-3. https://doi.org/10.1016/j.acci.2017.02.001

42. Unidad de salud de El Tiempo. Respiradores, una carrera vital para enfrentar el coronavirus. El Tiempo. $2020 \mathrm{Abr}$ 20. https://www.eltiempo.com/salud/respiradores-hay-suficientes-en-colombia-485904

43. CNN en español. Crisis en los hospitales por covid-19, ¿cuál es la ocupación de camas en tu país? https://cnnespanol.cnn.com/video/crisis-covid-coronavirus-camas-hospitales-perspectivas-bue nos-aires/?fbclid=IwAR0RaISWXS1F2J26vv3gNFhfTmNJzSkrQqINcLjYTYx2O40EozdgwC7Cx jg

44. Colombia. Ministerio de Salud y Protección Social. Resolución 1877 de 2013, mayo 30, por medio de la cual se efectúa la categorización del riesgo de las Empresas Sociales del Estado del nivel territorial para la vigencia 2013. Bogotá: El Ministerio; 2013.

45. Colombia. Ministerio de Salud y Protección Social. Resolución 2090 de 2014, mayo 29, por la cual se efectúa la categorización del riesgo de las Empresas Sociales del Estado del nivel territorial para la vigencia 2014 y se dictan otras disposiciones. Bogotá: El Ministerio; 2014. 
46. Colombia. Ministerio de Salud y Protección Social. Resolución 1893 de 2015, mayo 29, por la cual se efectúa la categorización del riesgo de las Empresas Sociales del Estado del nivel territorial para la vigencia 2015. Bogotá: El Ministerio; 2015.

47. Colombia. Ministerio de Salud y Protección Social. Resolución 2184 de 2016, mayo 27, por la cual se efectúa la categorización del riesgo de las Empresas Sociales del Estado del nivel territorial para la vigencia 2016 y se dictan otras disposiciones. Bogotá: El Ministerio; 2016.

48. Colombia. Ministerio de Salud y Protección Social. Resolución 1755 de 2017, mayo 26, por la cual se efectúa la categorización del riesgo de las Empresas Sociales del Estado del nivel territorial para la vigencia 2017 y se dictan otras disposiciones. Bogotá: El Ministerio; 2017.

49. Colombia. Ministerio de Salud y Protección Social. Resolución 2249 de 2018, mayo 30, por la cual se efectúa la categorización del riesgo de las Empresas Sociales del Estado del nivel territorial para la vigencia 2018 y se dictan otras disposiciones. Bogotá: El Ministerio; 2018.

50. Colombia. Ministerio de Salud y Protección Social. Resolución 1342 de 2019, mayo 29, por la cual se efectúa la categorización del riesgo de las Empresas Sociales del Estado del nivel territorial para la vigencia 2019. Bogotá: El Ministerio; 2019.

51. Colombia. Supersalud. Base de datos Entidades Liquidadas. [Consultado 2020 Jul 3] https://docs.supersalud.gov.co/PortalWeb/MedidasEspeciales/Directorio\%20de\%20Entidades/L VFT02\%20ENT\%20LIQUIDADAS.pdf

52. Colombia. Ministerio de Hacienda. Informe de evaluación año 2019 - programas de saneamiento fiscal y financiero visibilizados de empresas sociales del estado en riesgo bajo medio o alto. [Consultado 2020 Sep 23] Disponible en: http://www.urf.gov.co/webcenter/ShowProperty?nodeId=\%2FConexio nContent\%2FWCC_CLUSTER-141892\%2F\%2FidcPrimaryFile\&revision=latestreleased

53. Tendencias de El Tiempo. Así se preparan los hospitales de campaña para enfrentar el covid-19. El Tiempo. 2020 Mar 23 [Consultado 2020 Sep 22] https://www.eltiempo.com/justicia/servicios/coron avirus-asi-se-preparan-los-hospitales-de-campana-475838

54. Minsalud. Registro Especial de Prestadores de Servicios de Salud (REPS). https://prestadores.minsalu d.gov.co/habilitacion/

55. Unidad de salud de El Tiempo. Hospitales del país se alistan para ola de enfermos por COVID-19. El Tiempo. 2020 Mar 28. https://www.eltiempo.com/salud/como-estan-los-hospitales-en-colombia-par a-atender-emergencia-por-coronavirus-478060

56. Envigado te informa. La primera etapa de expansión de hospitalización para pacientes COVID-19 de la sede Santa Gertrudis ya es una realidad. Envigado informa. 2020 Sep 9. https:/www.envigadoteinforma.gov.co/la-primera-etapa-de-expansion-de-hospitalizacion-para-p acientes-covid-19-de-la-sede-santa-gertrudis-ya-es-una-realidad/

57. Colombia. Presidencia de la República. Decreto 2194 de 2004, julio 8, establecer las condiciones y procedimientos para disponer de información periódica y sistemática que permita realizar el seguimiento y evaluación de la gestión de las instituciones públicas prestadoras de servicios de salud y evaluación del estado de implementación y desarrollo de la política de prestación de servicios de salud y su impacto en el territorio nacional, las cuales serán de aplicación y obligatorio cumplimiento para las instituciones públicas prestadoras de servicios de salud y las direcciones departamentales, municipales y distritales de salud. Bogotá: Presidencia; 2004. 
58. Consultorsalud. Deuda con hospitales públicos del país es de más de $\$ 5,4$ billones. clínicas https://con sultorsalud.com/deuda-con-hospitales-publicos-del-pais-es-de-mas-de-54-billones/

59. Franco A. Seguridad social y salud en Colombia: estado de la reforma. Rev Salud Pública. 2000;2(1):1-16. https://revistas.unal.edu.co/index.php/revsaludpublica/article/download/18768/198 $45 / 61754$

60. Marín G, Vargas G, Shaw, A. Atención materna en un ambiente de mercado de servicios de salud, Medellín, 2008-2009. Rev Gerenc Polit Salud. 2010;9(19):108-23. http://www.scielo.org.co/pdf/rg ps/v9n19/v9n19a08.pdf

61. Escobar J. Ley 100 de seguridad social: implicaciones bioéticas. En: Escobar J, Da Costa M, Jácome S, Málaga H, Maldonado C, Montt J, et al. (eds.), Bioética y justicia sanitaria, $2^{\mathrm{a}}$ ed. Bogotá: El Bosque; 1999.

62. Hernández M. El derecho a la salud en Colombia: Obstáculos estructurales para su realización. Rev Salud Pública. 2000;2(2):121-144. https://revistas.unal.edu.co/index.php/revsaludpublica/article/vie $\mathrm{w} / 18882 / 19840$

63. Manrique L, Eslava J. Auscultando la corrupción en la salud: definición y causas. ¿Qué está en juego? Rev Colomb Obs Ginecol. 2011;62(4):308-314. http://www.scielo.org.co/pdf/rcog/v62n4/v62n4a03 .pdf

64. Echeverry López M. Reforma a la salud y reconfiguración de la trayectoria de acceso a los servicios de salud desde la experiencia de los usuarios en Medellín, Colombia. Rev Gerenc Polit Salud. 2011;10(20):97-109. https://doi.org/10.11144/Javeriana.rgsp10-20.rsrt

65. Colombia. DANE. Información Regional. [Consultado 2019 Sep 8] https://www.dane.gov.co/index.ph p/estadisticas-por-tema/informacion-regional

66. Gutiérrez C. El sistema de salud colombiano en las próximas décadas: cómo avanzar hacia la sostenibilidad y la calidad en la atención. Bogotá: Fedesarrollo; 2018.

67. Organización Panamericana de La Salud, Oficina Sanitaria Panamericana, Oficina Regional de la Organización Mundial de La Salud. Informe Dawson. sobre el futuro de los servicios médicos y afines 1920. Publicación Científica No. 93. Gran Bretaña: OPS y OMS; 1964.

68. Macinko J, Montenegro H, Nebot Adell C, Etienne C y Grupo de Trabajo de Atención Primaria de Salud de la Organización Panamericana de la Salud. La renovación de la atención primaria de salud en las Américas. Rev Panam Salud Pública. 2007;21(2/3):73-84. https://www.paho.org/hq/dmdocuments/ 2010/Renovacion_Atencion_Primaria_Salud_Americas-OPS.pdf

69. Colombia. Ministerio de salud y protección social. Resolución 2626, septiembre 27, por el cual se modifica la Política de Atención Integral en Salud - PAIS y se adopta el Modelo de Atención Integral Territorial - MAITE. Bogotá: El Ministerio; 2019. 
El estado de la red pública hospitalaria en Colombia para enfrentar el

COVID-19, posterior a la política de categorización de riesgo fiscal y financiero

Notas

1 Que corresponden a: Empresas Promotoras de Salud (EPS), Empresas Promotoras de Salud del Régimen Subsidiado (EPSS), Entes Territoriales Departamentales y municipales, Administradoras de Riesgos Profesionales, Fondo de Solidaridad y Garantía, Fondos Locales de Salud, etc. 